\section{On the Risk of Introduction of an Emerging Infectious Disease}

\author{
Marcos Amaku \\ José H.H. Grisi-Filho \\ Raul Ossada \\ Fernando Ferreira \\ Faculdade de Medicina Veterinária e Zootecnia da Universidade de São Paulo \\ - USP.
}

Corresponding Author: Marcos Amaku. Faculdade de Medicina Veterinária e Zootecnia da Universidade de São Paulo. Av. Prof. Dr. Orlando Marques de Paiva, 87 - CEP 05508-270, São Paulo, SP Brasil. E-mail: amaku@usp.br
Dear Editors,

An interesting editorial ${ }^{1}$ published in Revista Brasileira de Epidemiologia addresses the threat of an influenza pandemic and mentions "the information technology that makes it possible to monitor the distribution of new cases in real time". We are aware that the risk of spread of an emerging infectious disease from one country to another depends on the number of travelers and on the ability of the monitoring system to detect infected passengers. In this letter, we describe a quantitative methodology to estimate the risk of introduction of an emerging infectious disease into a diseasefree country through the movement of persons between countries in international flights. Our aim is to contribute to the discussion about the importance of factors that influence the spread of influenza and other emerging diseases.

Let us suppose that $n$ travelers depart from country $A$ to country $B$, each of which with a probability $p$ of being infected by an emerging infectious disease occurring in $A$. The proportion of infected persons (population prevalence) in A would give a rough estimate for probability $p$. An additional assumption is that the probability $p$ that a passenger is infected does not depend on the probability that another passenger gets infected in the country of origin. Approximately, there is a probability $(1-p)^{n}$ that all $n$ persons are not infected. Therefore, the probability of introduction of at least one infected person in the destination country $B$ may be estimated as $1-(1-p)^{n}$. This would be the risk of disease introduction in the absence of an active surveillance system to detect disease.

Assuming that it is possible to estimate a sensitivity $S e$ and a specificity $S p$ for the surveillance system in the airport customs, then the probability of introduction of a healthy (true negative) person is equal to the negative predictive value, $N P V$. That is, the probability of introduction of $n$ healthy persons is $(N P V)^{n}$. Hence, the probability of introduction of at least one infected person is 


$$
1-(N P V)^{n}=1-\left[\frac{(1-p) S p}{S p(1-p)+p(1-S e)}\right]^{n}
$$

Similar approaches to the model presented here have been applied to data for severe acute respiratory syndrome (SARS) ${ }^{2}$ and to risk assessment for animal import ${ }^{3}$. To illustrate the usefulness of this method, Figure 1 shows the results of some calculations using the above equation for $p=10^{-6}, S p=1.0$, and some values of $S e$. The risk of disease introduction increases as $n$ increases, and decreases as $S e$ increases. Note that, for $S p<1.0$ or for values of $S e$ lower than those used in the calculations, risk estimates would be even greater than the values observed in Figure 1.

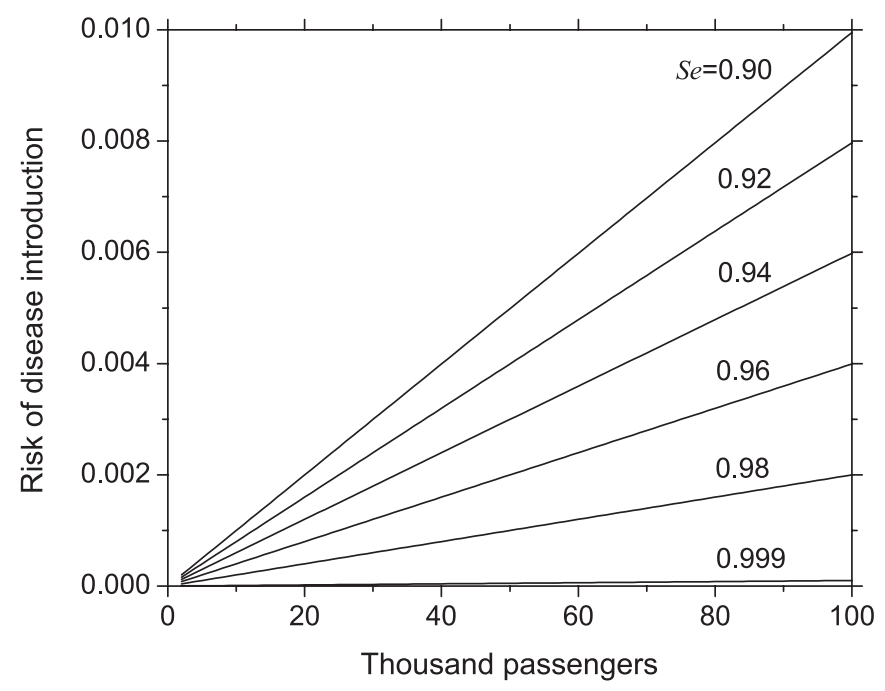

Figure 1 - Probability of disease introduction for $p=10^{-6}, S p=1.0$, and different values of Se (numbers over the lines), as a function of the number of passengers.

Figura 1 - Probabilidade de introdução da doença para $p=10^{-6}, S p=1,0$, e valores diferentes de Se (números sobre as linhas), como uma função do número de passageiros.

It is difficult to obtain a reliable estimate of $p$ in a timely manner. We assume that, based on data on the previous occurrence of similar diseases and/or on expert opinion, it is possible to estimate an upper bound for the probability $p$ of a traveler being infected (worst scenario). We then may estimate the risk of introduction of at least an infected person quantitatively, on the basis of the influx of travelers from another country.

It is possible to extend the preceding equation to estimate the risk considering the influx of passengers from several countries, by multiplying $\left(N P V_{i}\right)^{n_{i}}$ for each country $i$ and subtracting the result from 1 , obtaining

$$
1-\left[\frac{\left(1-p_{1}\right) S p}{S p\left(1-p_{1}\right)+p_{1}(1-S e)}\right]^{n_{1}} \times\left[\frac{\left(1-p_{2}\right) S p}{S p\left(1-p_{2}\right)+p_{2}(1-S e)}\right]^{n_{2}} \times \ldots
$$

in which $n_{i}(i=1,2, \ldots)$ is the number of travelers arriving from a country with prevalence $p_{i}(i=1,2, \ldots)$.

The sensitivity of a surveillance system may be estimated based on the number of non-detected infected persons (i.e. false negatives) entering the country during recent pandemics, such as the influenza virus $\mathrm{A}(\mathrm{H} 1 \mathrm{~N} 1)^{4}$. The disease incubation and infectious 
periods are factors that affect the sensitivity of the surveillance system. False positives (e.g., persons infected with diseases with similar symptoms) may be detected, resulting in a certain specificity. Estimates for the sensitivity and specificity of screening tests, such as thermal scanners used in airports, will probably be available in the future.

Given estimates for $S e$ and $S p$, the risk will be a function of the expected number of travelers arriving in a period. Estimates for the risk of disease introduction may help public health officials to decide whether or not to screen passengers arriving at the beginning of an outbreak. Furthermore, depending on $S e$ and $S p$, screening tests may lower the risk of an early introduction of a higher number of infected persons.

\section{References}

1. Carvalheiro JR. Popular pandemic. Rev Bras Epidemiol 2009; 12(2): 101-4.

2. Dell'Omodarme M, Prati MC. The probability of failing in detecting an infectious disease at entry points into a country. Statist Med 2005; 24: 2669-79.

3. Murray N. Handbook on import risk analysis for animals and animal products. Vol. 2: Quantitative risk assessment. Paris: World Organisation for Animal Health (OIE); 2004.
4. World Health Organization. Influenza A (H1N1). Available from http://www.who.int/ csr/disease/ swineflu/en/index.html [Acessed on 05 June 2009].

Recebido em: 27/08/09 Versão final reapresentada em: 09/06/10 Aprovado em: 21/06/10 